\title{
Insights into the Formation Mechanism of CdSe Nanoplatelets Using in situ X-ray Scattering
}

Nicolo Castro, ${ }^{\dagger}$ Cécile Bouet, ${ }^{\ddagger}$ Sandrine Ithurria, ${ }^{\ddagger}$ Nicolas Lequeux, ${ }^{\ddagger}$ Doru Constantin, ${ }^{\dagger}$ Pierre Levitz, ${ }^{\complement}$ Diego Pontoni, ${ }^{\S}$ and B. Abécassis* ${ }^{*} \|, \perp$

$\dagger$ †aboratoire de Physique des Solides, CNRS, Univ. Paris-Sud, Université Paris-Saclay, 91405 Orsay Cedex, France

$\ddagger$ Laboratoire de Physique et d'Etude des Matériaux, ESPCI-Paris, PSL Research

University, Sorbonne Université, CNRS, 10 rue Vauquelin, 75005, Paris, France

ФSorbonne Université, CNRS, Physico-Chimie des Électrolytes et Nanosystèmes Interfaciaux, PHENIX, F-75005 Paris, France

$\S$ Partnership for Soft Condensed Matter (PSCM), ESRF - The European Synchrotron, 71 Avenue des Martyrs, 38043 Grenoble, France

||Laboratoire de Chimie, CNRS, École Normale Supérieure de Lyon, 46 allée d'Italie, 69364 Lyon, France

$\perp$ Laboratoire de Physique des Solides, CNRS, Univ. Paris-Sud, Université Paris-Saclay, 91405 Orsay, France

E-mail: benjamin.abecassis@ens-lyon.fr

\begin{abstract}
Two dimensional ultra thin CdSe nanoplatelets have attracted a large interest due to their optical properties but their formation mechanism is not well understood. Several
\end{abstract}


different mechanisms have been proposed: confined growth in a surfactant mesophase acting as a template, anisotropic ripening of small seeds into 2D nanoplatelets or continuous anisotropic growth of a limited number of nuclei. However, quantitative in situ data that could validate or disprove these formation scenarios are lacking. We use synchrotron-based small-angle and wide-angle X-ray scattering to probe the formation mechanism of CdSe nanoplatelets synthesized using a heating-up method. We prove the absence of a molecular mesophase in the reactive medium at the onset of nanoplatelet formation ruling out a templating effect. We also show that our data are inconsistent with the anisotropic ripening of small seeds whereas the evolution of the SAXS patterns during the reaction is consistent with the continuous lateral growth of nanoplatelets fed by reactive monomers. Finally, we show that when the final temperature of the synthesis is lowered, nanoplatelets with larger lateral dimensions form. We unravel that they bend in solution during their growth to yield nanoscrolls.

\section{Keywords}

CdSe,semi-conducting nanoplatelets, quantum dots, quantum well 


\section{Introduction}

Semi-conducting nanoplatelets (NPLs) are two-dimensional nanoparticles where quantum confinement only occurs along their thickness. ${ }^{1,2}$ Discovered ten years ago, they have been the subject of intense research ever since due to their outstanding optical properties such as very fast narrow fluorescent emission, ${ }^{3}$ high quantum yields, fast Föster Resonant Energy Transfer which even out-paces Auger recombination ${ }^{4}$ or highly polarized emission. ${ }^{5-7}$ Synthetic control over these 2D structures has been extended to core-crown, core-shell structures and other materials of the II-VI families have been obtained. ${ }^{8,9}$ In the case of zinc-blende NPLs, their thickness is perpendicular to the [001] direction and the top and bottom planes are $\mathrm{Cd}$ rich facets on which surface ligands are adsorbed. NPLs thickness can be controlled at the atomic level and depending on the synthesis condition, they can be 2, 3, 4 or 5 CdSe monolayers (ML) thick where $N$ monolayers correspond to $N+1$ planes of Cd alternated with $N$ planes of Se. Direct synthesis of thicker (from 6 to 9 ML) NPL using chloride salts have recently been reported. ${ }^{10,11}$ The synthesis of NPLs usually involves a long-chain cadmium carboxylate which reacts with a selenium precursor. Until recently, it was thought that a second shorter chain ligand was necessary to induce anisotropic growth and most of the current synthetic protocols include cadmium acetate as a secondary cadmium source. Variations in the synthetic conditions enables some control over the thickness and lateral dimensions of the NPLs. 2 and 3 ML NPLs are more likely to be formed at temperatures between 150 and $200^{\circ} \mathrm{C}$ while higher temperatures in the $200-250^{\circ} \mathrm{C}$ range favour the formation of thicker 4 and 5 ML NPLs. In some cases, the NPLs are curved. Large 3 ML NPL have been shown to roll into nanoscrolls under the effect of ligand induced surface stress when they reach sufficiently large lateral dimensions. ${ }^{12,13}$ Twisted shapes have also been observed in 5 ML NPLs upon addition of oleic acid and a mechanism with similar physical origin is likely. ${ }^{14}$

The formation mechanism of these NPLs is not well understood yet. The zinc-blende crystallographic structure of the NPLs does not display any symmetry break which would 
endow one facet with a larger surface energy, thus slowing down its growth at the expense of other more stable crystal planes. Several competing mechanisms can be proposed to explain the formation of these $2 \mathrm{D}$ structures. The first mechanism that has been proposed is the continuous transition between small seeds and NPLs through lateral extension. ${ }^{15}$ In this scenario, a given number of nuclei are formed at the beginning of the reaction and they later grow in a 2D fashion through the addition of monomers in solution. Another hypothesis is the presence of an organic molecular template which would confine the growth of the particles in a limited space. This idea has been proposed for metallic nanoparticles and wurtzite CdSe nano-ribbons have been shown to obey such a formation pattern. ${ }^{16,17}$ However, in the case of zinc-blende NPLs, no direct in situ evidence has been provided so far to support this mechanistic pathway. Moreover, it has recently been shown that NPL could be synthesized without any mesophase present, calling into question the universality of this mechanism. ${ }^{18}$ Another pathway that can yield two dimensional particles is anisotropic ripening of small seed clusters. In this scenario, small nanoparticles are first formed in solution and they later assemble into 2D NPLs at constant volume fraction in crystalline material. The driving force for the ripening to occur in $2 \mathrm{D}$ instead of isotropically is a preferred configuration of the passivating ligands at the nanocrystal/solution interface. When the nanocrystals attach one to each other to yield platelets the ligand monolayer at the top and bottom faces of the NPL densifies. This maximizes the attractive van der Waals interactions between the alkyl chains and results in a net energy gain with compared to 3D growth. ${ }^{19} 5 \mathrm{ML}$ NPLs were synthesized using this pathway recently: ${ }^{20}$ carboxylate coated seeds were first synthesized and their ripening at high temperature in the presence of cadmium acetate and oleic acid afforded well defined NPLs with lateral dimensions ranging from 10 to $40 \mathrm{~nm}$. A recent paper also suggested that anisotropic ripening could occur at the purification stage when solvents that strip ligands at the surface of nanoparticles are used. ${ }^{21}$

In an important contribution, Riedinger et al. have used experiments, kinetic modelling, 
DFT and kinetic Monte-Carlo simulations to evidence a kinetic growth instability which can explain the formation of zinc-blende NPLs. ${ }^{18,22}$ This mechanism can occur when the concentration in reactive monomers is sufficiently large for the limiting process of the growth to be the reaction at the nanocrystal/solution interface. In this case, 2D growth can be energetically preferred over isotropic growth since $2 \mathrm{D}$ growth of NPL at edges is much faster than the growth of large facets. This model is consistent with a number of experimental facts such as the preferred formation of thick NPLs as temperature increases, the stability of different NPLs thicknesses depending on the material type or the ripening of thin NPLs into thicker ones as the temperature is increased. The model does not make any assumptions on the type of surfactant used for the synthesis. It has been thought for some time that the presence of two surfactants, one with a long and another with a short alkyl chain was a necessary condition for the formation of NPLs. This rationale was based on the synthesis conditions which always included these two types of cadmium carboxylate. However, Riedinger et al. ${ }^{18}$ have shown that CdSe NPLs could be obtained in the melt of cadmium carboxylate without the use of short chain ligands. They explained that adding a short chain carboxylate decrease the solubility of the salt and thus increases the local concentration in monomers. This enables the kinetic instability to occur under reaction controlled conditions whereas in the case of soluble precursors this regime can not be attained. Recently, the possibility to synthesize NPLs without short chain carboxylate has been confirmed using cadmium octanoate. ${ }^{23}$ However, no trace of insoluble material was visible with the naked eye at the onset of NPLs formation. To sum up the current understanding of NPLs formation, several different mechanisms have been proposed to date but direct experimental data are lacking to prove or disprove them. Mechanisms are mostly inferred from ex-situ and/or post synthesis electron microscopy analysis and UV VIS spectroscopy.

Small-Angle X-ray scattering (SAXS) $)^{24,25}$ and wide angle X-ray scattering (WAXS) are suitable techniques to probe the structure of materials at scales ranging from the $\AA$ to $\mu \mathrm{m}$. 
With the brilliance of the X-ray beam available at third generation synchrotrons, it is now possible to probe reacting media with a time resolution better than $100 \mathrm{~ms}$. These in situ studies have proven insightful for various physico-chemical phenomena at the nanoscale ${ }^{26}$ such as nucleation, growth and self-assembly of metallic or oxyde nanoparticles ${ }^{27-30}$ and the Kirdendall effect. ${ }^{31}$

Concerning more specifically metal chalcogenides, SAXS has already been used for the sizing of quantum confined nanoparticles ${ }^{32-35}$ but reports on time-resolved experiments are scarcer. We have recently shown that nucleation and growth of CdSe nanoparticles could be probed in-situ using time-resolved SAXS/WAXS. ${ }^{36}$ Van der Staam et al. ${ }^{37}$ have used the same techniques to provide mechanistic insights into the nucleation and growth of $\mathrm{Cu}_{2-x} \mathrm{~S}$ nanoplatelets. They showed that the anisotropic growth proceeds through a templated mechanism where $\mathrm{Cu}$-thiolate complexes self-assemble into lamellar structures which remain present during the formation of the nanoparticles and guide their growth through the confinement of mass transport and the prevention of their inter-layer sintering.

Here we provide the first experimental data on the formation of 3 ML CdSe NPLs using a heating up procedure. The NPLs are synthesized using a one pot method where a mixture of cadmium oleate and cadmium acetate are mixed with Trioctylphosphine-Se (TOP-Se) and heated towards a temperature comprised between 170 and $200^{\circ} \mathrm{C}$. The SAXS and WAXS patterns are acquired during NPL formation. Our data show that no template is present during the nucleation and growth of the NPLs which is proven by a clear $q^{-2}$ regime at intermediate wave-vectors. We also do not detect the presence of small magic size clusters acting as transient species prior to anisotropic ripening. Both the SAXS and the WAXS sequences show a gradual lateral extension of the NPLs which grow from monomers in solution. In some experimental conditions, we show that the NPLs bend during their growth with a radius of curvature which is the same as the one observed ex situ. 


\section{Results and discussion}

A)

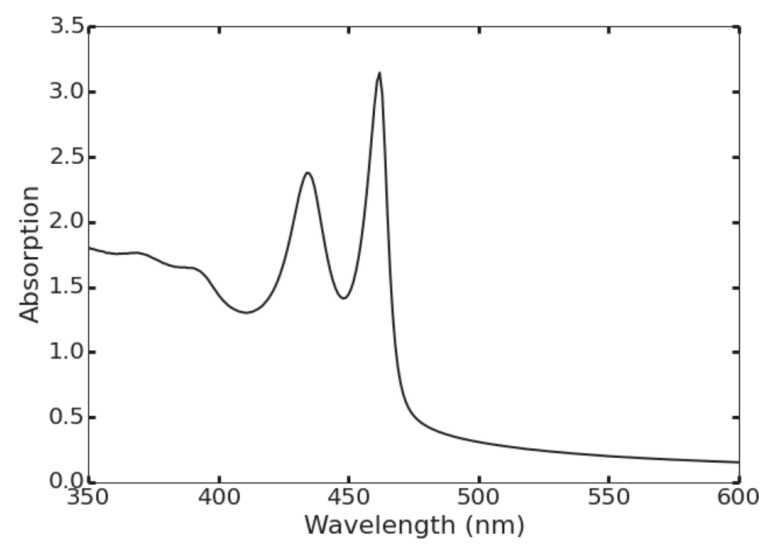

C)

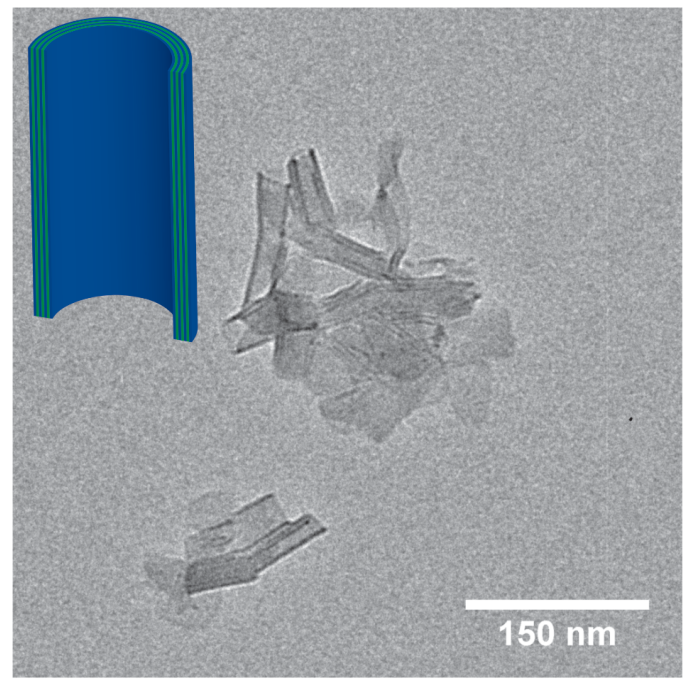

B)

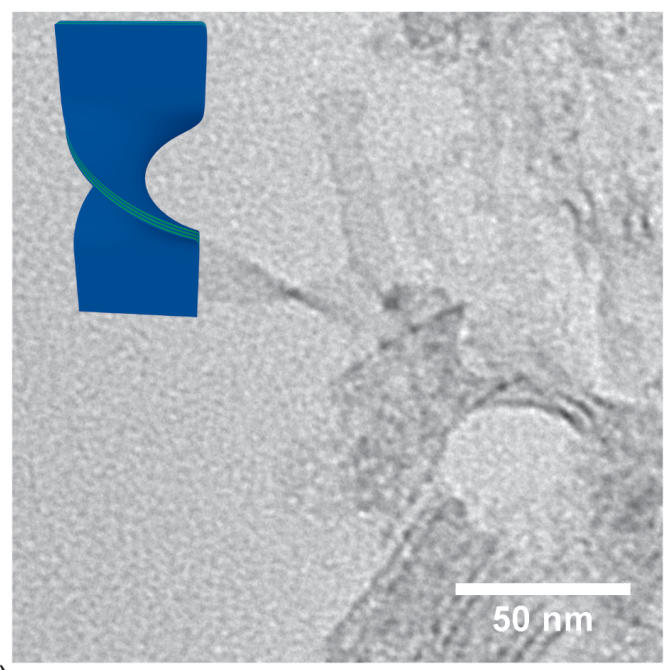

D)

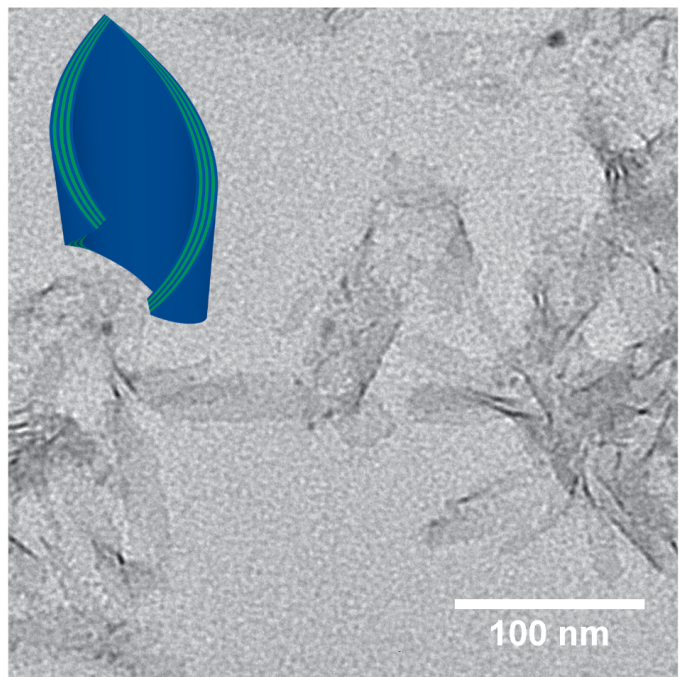

Figure 1: A) UV VIS spectra of CdSe NPLs synthesized through heat-up synthesis. B-D) TEM images of the CdSe NPLs showing the different bending/twisting configurations.

Most of the previous syntheses of zinc-blende CdSe NPLs rely on the injection of cadmium acetate during the heating of a mixture of selenium powder and cadmium carboxylate bearing long aliphatic chains. This hot injection protocol, combined with the high synthesis temperature $\left(200\right.$ to $\left.240^{\circ} \mathrm{C}\right)$, is difficult to reconcile with current in situ probing set ups. To solve this issue, we worked out a heat-up synthesis of CdSe NPLs which relies on prior reaction of cadmium acetate with oleic acid in octadecene at $150^{\circ} \mathrm{C}$ (see supplementary for 
further details). During this phase, oleic acid reacts with cadmium acetate yielding an acetate/oleate complex with formula $\mathrm{Cd}(\text { oleate })_{2-\mathrm{x}}(\text { acetate })_{\mathrm{x}}$. A certain volume of this solution is diluted in octadecene and TOP-Se is added at ambient temperature. The solution is then heated to the desired temperature. Characterization of the obtained product is displayed in Figure 1. The absorption UV-VIS spectrum displays two peaks at 462 and $435 \mathrm{~nm}$ characteristic of the excitonic features of 3 monolayers (3ML) thick CdSe nanoplatelets. These NPLs consist of two and a half CdSe unit cells in their thickness i.e. 4 Cd atoms and 3 Se atoms. Two smaller peaks at shorter wavelengths (below $400 \mathrm{~nm}$ ) indicate the presence of a small quantity of 2 ML thick NPLs. Electron microscopy confirms the synthesis of thin NPLs. Most of them are not flat. We notice that they are rolled along a cylinder or rotate along a diagonal. The radius of the cylinders is homogeneous and comprised between 12 and $14 \mathrm{~nm}$ as already described. ${ }^{12,13}$ In some cases, we also observe twisted NPLs which adopt a bow-like shape. In this case, the center of the particle appears darker.

To probe the formation mechanism of these NPLs in situ, we performed SAXS/WAXS time-resolved experiments on the ID02 beamline at $\mathrm{ESRF}^{38}$ and used the same experimental set up as described in ref. ${ }^{36}$ The reaction media comprising the cadmium precursor described previously and TOP-Se in octadecene is introduced in a glass capillary which is then mounted on a heating stage enabling SAXS and WAXS acquisition during the heating of the solution. To make sure that the reaction in the capillary gave the same results as in a laboratory flask where the volume of the reaction is much larger, we checked by UV-VIS and TEM that the two methods yielded qualitatively similar results.

We start to examine qualitatively the sequence of SAXS patterns for a temperature ramp going from 30 to $200{ }^{\circ} \mathrm{C}$ at $1{ }^{\circ} \mathrm{C}$ per second at wave-vectors between 0.073 and $4.53 \mathrm{~nm}^{-1}$ corresponding to distances comprised between 1 and $90 \mathrm{~nm}$ in real space. Figure 2.A shows the evolution of the SAXS patterns during the first 105 seconds. At low temperature, we observe a $\mathrm{q}^{-4}$ regime at low $\mathrm{q}$ followed by a plateau and a slow decrease at high $q$ with a 

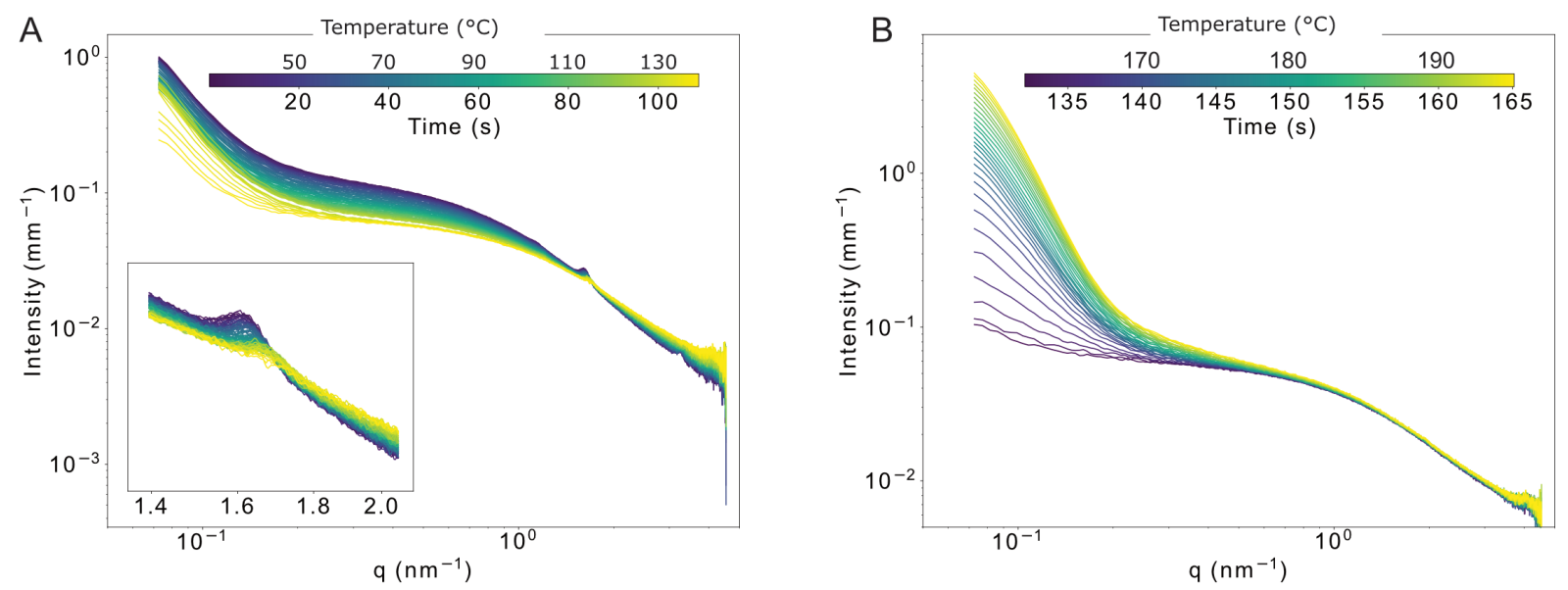

C
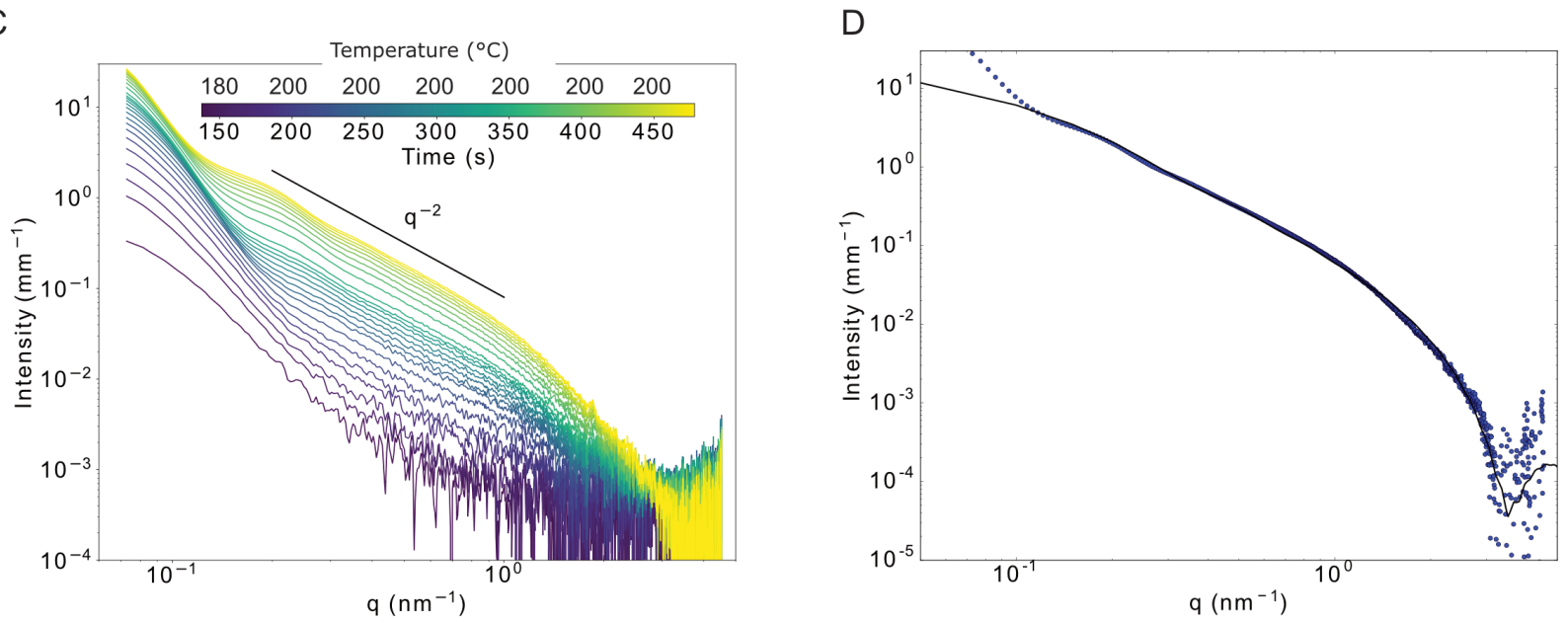

Figure 2: Sequences of SAXS patterns during the formation of CdSe NPLs with a final temperature of $200{ }^{\circ} \mathrm{C}$. A) Between 0 and $105 \mathrm{~s}$ corresponding to 30 to $135^{\circ} \mathrm{C}$. The intensity at low q decreases while the scattering peak at $1.63 \mathrm{~nm}^{-1}$ gradually disappears. B) Between 125 and $164 \mathrm{~s}$ corresponding to 155 to $200^{\circ} \mathrm{C}$. The intensity at low q increases showing the onset of nanoparticle formation. C) Between 165 and $450 \mathrm{~s}$ at $200{ }^{\circ} \mathrm{C}$, after subtraction of the SAXS intensity at $\mathrm{t}=120 \mathrm{~s}$ representing the SAXS background from the reaction precursors (see main text). Note the $q^{-2}$ slope showing the 2D character of the scattering objects. D) SAXS pattern of the reaction product at the end of the experiment for a final temperature of $200^{\circ} \mathrm{C}$ and theoretical SAXS intensity for a parallelepiped with dimensions $10 \times 10 \times 0.9$ $\mathrm{nm}$. 
broad peak at $\mathrm{q}=1.63 \mathrm{~nm}^{-1}$. These features can be explained by the $\mathrm{Cd}$ precursor being not fully solubilized in the solvent. The $q^{-4}$ slope corresponds to the Porod regime of large $\mathrm{Cd}(\text { oleate })_{2-x}$ (acetate $)_{x}$ domains which segregate in solution and thus display scattering contrast. These domains are larger than $100 \mathrm{~nm}$ since we do not reach their Guinier regime with our SAXS configuration which probes a maximum length scale of $2 \pi / q_{\min }=94 \mathrm{~nm}$. The peak at high $q$ is caused by the partial crystallization of the alkyl chains within these domains. As temperature increases, the intensity at low q decreases and the peak disappears, while the intensity at high $\mathrm{q}$ increases. These data indicate that the some precursor solubilize as small micelles in the solvent as temperature increases similarly to what had been observed for cadmium myristate in our previous study. ${ }^{36}$

After $120 \mathrm{~s}$ (corresponding to $150^{\circ} \mathrm{C}$ ), the intensity at low $q$ increases sharply, gaining one order of magnitude at $0.072 \mathrm{~nm}^{-1}$ while remaining almost constant for $\mathrm{q}>0.6 \mathrm{~nm}^{-1}$ (Figure 2.B). Such an increase of the intensity at low $q$ indicates the formation of nanometric objects in solution alongside precursor micelles which dominate the signal at higher wave vectors.

In order to get insight on the shape of these scattering objects, we subtract the SAXS pattern at $\mathrm{t}=120 \mathrm{~s}$ from the subsequent ones (Figure 2.C and D). The resulting SAXS patterns can be divided in three different parts. At $\mathrm{q}>2 \mathrm{~nm}^{-1}$, since the signal of the micelles dominates, the subtracted patterns are noisy and it is not possible to extract valuable information from this part of the curves. At intermediate q-range, we note the emergence of $\mathrm{a}^{-2}$ regime whose extension grows towards smaller wave-vectors with time. At smaller $\mathrm{q}$, the intensity is increasing with a slope larger than 2. In small angle scattering, the slope of the $I$ vs $q$ curve in log-scale is indicative of the shape of the scattering object. A q $\mathrm{q}^{-1}$

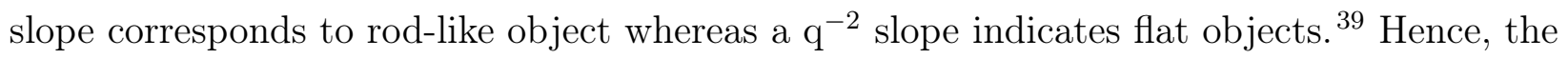
intermediate q-range with the $\mathrm{q}^{-2}$ slope is a non-ambiguous proof of the appearance of flat, plate-like objects in solution. Moreover, its extension towards smaller wave vectors as the reaction proceeds shows that the nanoplatelets lateral size is increasing with time.

To further prove the plate-like nature of the objects, we compare the SAXS pattern at the 
end of the reaction to the theoretical form factor of a $10 \times 10 \times 0.9 \mathrm{~nm}$ parallelepiped (Figure 2.D). The agreement between the data and the theoretical model is excellent. Both the slope at intermediate $\mathrm{q}$ and the high q regime are well reproduced. We note, however, a discrepancy at lower $q$ where the experimental intensity is higher than that of the model. This prevents a reliable measurement of the lateral extension of the NPLs and $10 \mathrm{~nm}$ represents a lower bound value. We will discuss this upturn in the low q range later.

This SAXS sequence can rule out two formation pathways. First, at the onset of particle formation, there is no evidence of a molecular template which would confine the growth of the NPLs in two dimensions. Mesophases such as lamellar structures would show up in the SAXS pattern with diffraction peaks spaced evenly which is obviously not the case here. Hence, in contrast with wurtzite nanoplatelets which are formed in a lamellar phases of alkylamines, a template pathway can be ruled out for these zinc-blende nanoplatelets. This confirms previous findings ${ }^{18,23}$ in a more direct and unambiguous way.

It has recently been suggested that nanoplatelets were formed at the purification level during which small nanoclusters assemble to yield anisotropic platelets. ${ }^{21}$ The in situ experiments carried out in our experimental conditions rule out this mechanism since flat nanoplatelets are already detected in the reacting medium prior to purification with an antisolvent. It is also possible that nanoplatelets form via ripening of small magic sized clusters previously formed in solution. These clusters would be visible in SAXS but since the scattering power scales like the volume squared, their SAXS signal would be much smaller than the one of much bulkier nanoplatelets. We can however calculate orders of magnitudes to see if this hypothesis is credible. At the end of our sequence, we saw that the signal is close to that of NPLs with $10 \times 10 \mathrm{~nm}$ lateral dimensions and a thickness $(t)$ of $0.9 \mathrm{~nm}$. Since the intensity scattered at low $\mathrm{q}$ is around $10 \mathrm{~mm}^{-1}$, this SAXS signal corresponds to a volumic fraction of NPLs: $\Phi_{p}=I_{p}(0) /(S t \Delta \rho)$ where $S$ is the surface and $\Delta \rho$ is the scattering length density contrast between CdSe and the solvent. If the same quantity of material was previously present in solution in the form of CdSe magic size clusters of $1 \mathrm{~nm}$ radius $\left(R_{c}\right)$, 
this would yield an almost flat signal over the whole q-range with intensity $\simeq I_{p}(0) \times \frac{4 / 3 \pi R_{c}^{3}}{S t}$ i.e. around $0.42 \mathrm{~mm}^{-1}$. Such an intense signal is orders of magnitude larger than the limit of detectability of our experiment and even a tiny fraction of it would be visible. On the contrary, the first detectable signal features a clear $\mathrm{q}^{-2}$ slope which rules out a formation mechanism where a significant quantity of CdSe is first produced in the form of small clusters which ripen afterwards.

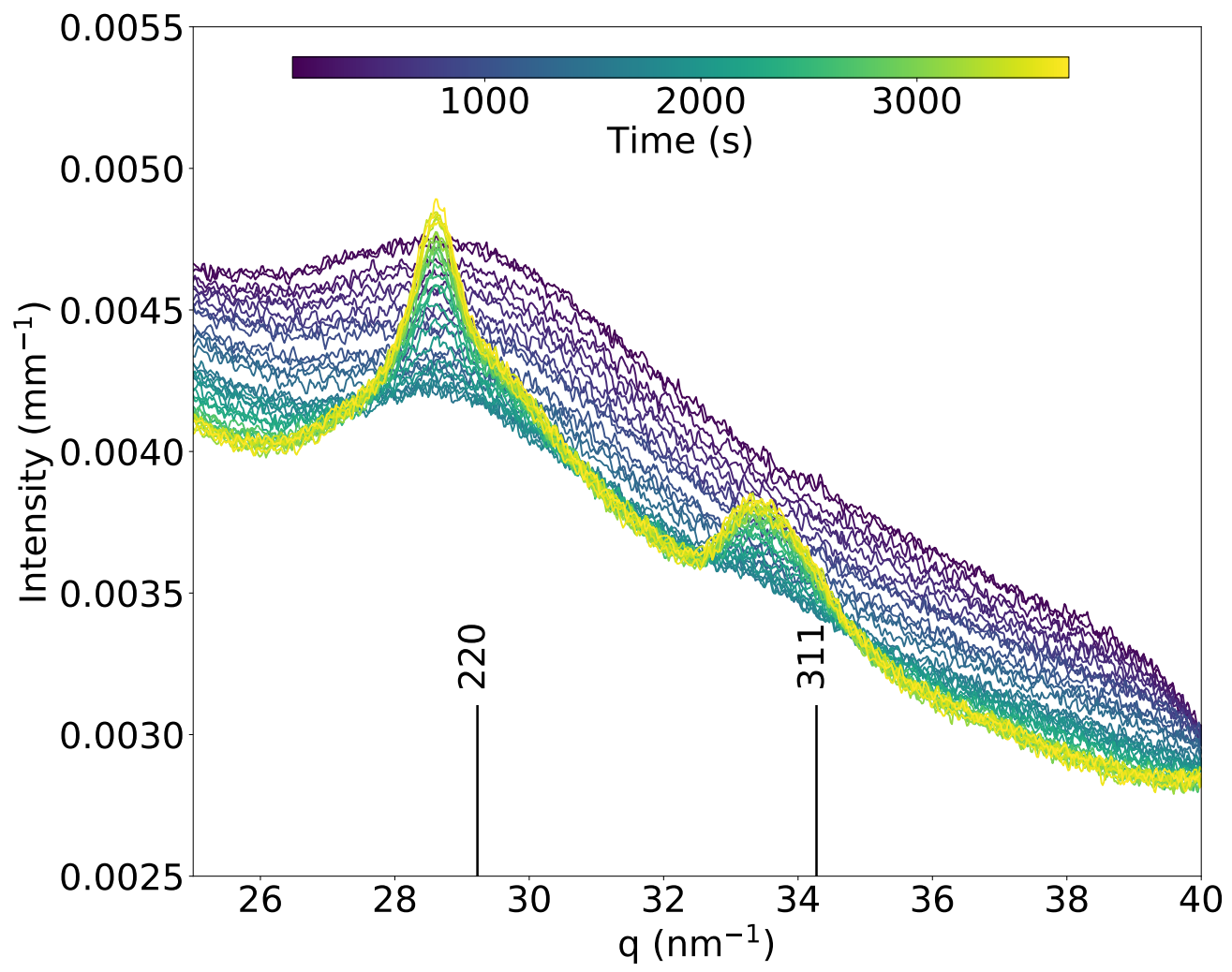

Figure 3: Sequence of WAXS patterns during the formation of CdSe NPLs over the course of the reaction from ambient temperature to $200^{\circ} \mathrm{C}$. Two peaks corresponding to the $(220)$ and (311) reflections of the zinc-blende structure are clearly visible.

Figure 3 shows the wide angle X-ray scattering patterns recorded during the NPLs formation. During the first $100 \mathrm{~s}$, no peak is observed and the signal corresponds to that of the solvent and precursors. As the reaction proceeds and the NPLs appear, the (220) and (311) peaks develop at respectively 29.2 and $34.3 \mathrm{~nm}^{-1}$ and their intensity increases. The (220) peak displays a shoulder at high angle due to the deformation of the lattice towards a 
tetragonal structure. As already noted, ${ }^{40-43}$ surface ligands at the basal planes of the NPLs induce a surface strain which deforms the crystal lattice from a cubic to a tetragonal unit cell. Thus, atomic planes which should be equivalent in diffraction are not equivalent any more depending on the orientation with respect to the basal planes. This is the case for the (220) peak where the (220) reflection splits into an actual (220) peak and a (202)/(022) peak at higher angles. This indicates that surface strains are already present during the formation of the NPLs and do not develop afterwards.

The (220) peak was fitted to a pseudo-Voigt function (figure S1) with a polynomial background for all the WAXS patterns in the sequence. The fits are excellent on the small q half of the peak but small deviations from the model are observed on the high q part of the peak due to the shoulder previously mentioned. Several relevant parameters were extracted from the fitted values: the peak area which is proportional to the amount of crystalline material, the peak position is compared to the bulk value and is converted into a lattice strain, the full-width at half maximum (FWHM) informs on the size of the crystallites via the Scherrer formula. As expected, the crystal volume fraction increases from 100 to $1000 \mathrm{~s}$. The position of the peak being shifted at lower q with respect to the bulk value, the crystal lattice is expanded. This is consistent with previous findings with carboxylate ligands. ${ }^{40,41}$ Interestingly, the magnitude of the strain increases as the reaction proceeds and goes from $2 \%$ after $200 \mathrm{~s}$ to $2.8 \%$ at the end of the reaction. Finally, the diffraction peak FWHM decreases with time showing that the crystallites are growing in size as the reaction proceeds. This is consistent with the SAXS data and comforts the scenario of a gradual extension of the NPLs with time.

In the same experimental conditions, but with a final temperature of $170{ }^{\circ} \mathrm{C}$ (instead of $200{ }^{\circ} \mathrm{C}$ ) we observed the same succession of mechanistic steps. First, solubilization of the precursor and disappearance of the high q peak (Fig 4.A.), then onset of anisotropic nanoparticle formation with a $\mathrm{q}^{-2}$ regime which extends towards smaller q (Fig 4.B. and C.). However, after these phases which are common to the two temperatures, we observe 
A
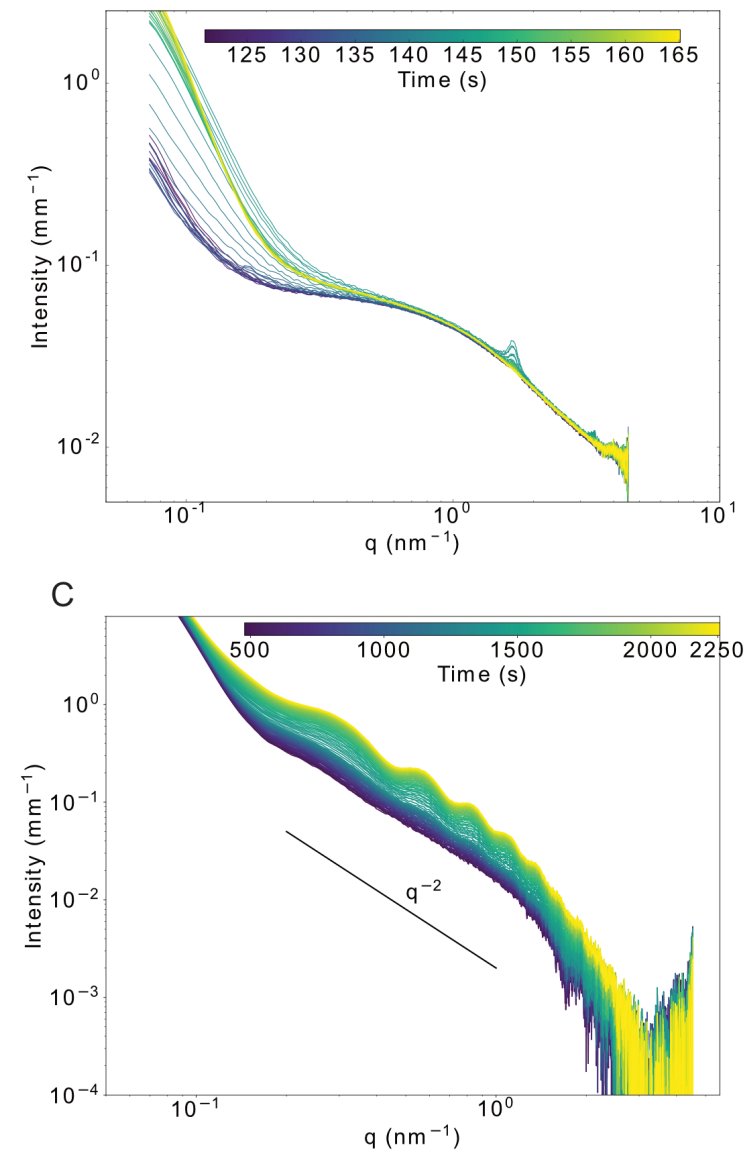

B

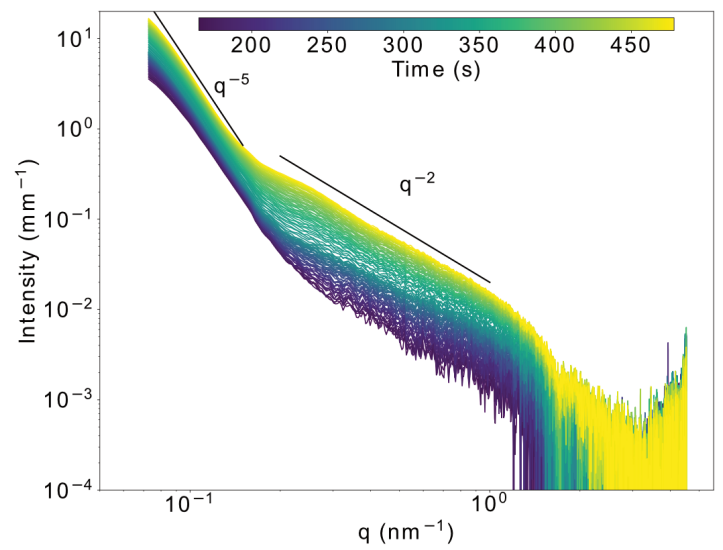

D

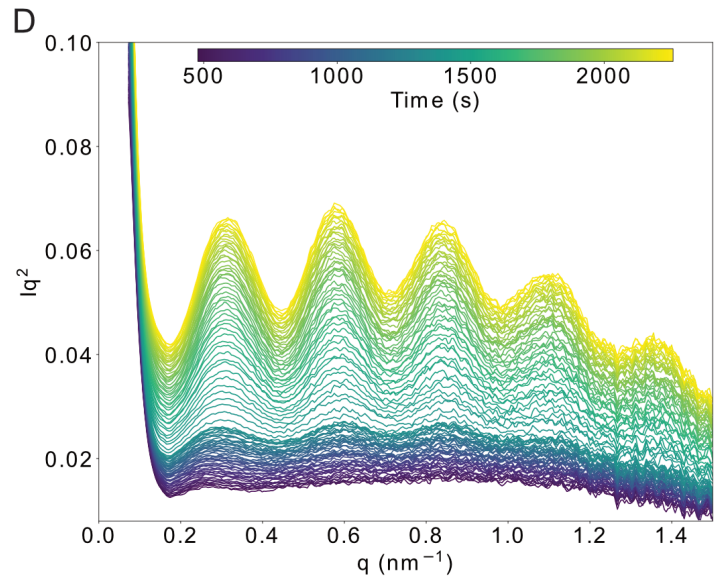

Figure 4: A) and B) Sequence of SAXS patterns between 120 and 500 seconds for a final temperature of $170^{\circ}$. Note the onset of $\mathrm{q}^{-2}$ regime at intermediate $\mathrm{q}$ range and the $\mathrm{q}^{-5}$ regime at small wave-vectors. C) SAXS patterns for times between 500 and $2250 \mathrm{~s}$. Oscillations at intermediate q-range are visible. D) Guinier plots for the SAXS patterns between 500 and $2250 \mathrm{~s}$. 
oscillations of the scattered intensity between 0.2 and $1.5 \mathrm{~nm}^{-1}$ for $170{ }^{\circ} \mathrm{C}$. These oscillations start to appear after 10 minutes of reaction and remain until the end of the sequence. They can be more easily visualized in the Guinier plot $\left(I \times q^{2}\right.$ vs $\left.q\right)$ shown on figure 4.D. We note that the position of these oscillations slightly shifts towards larger $q$ with time. For example, the maximum of the first oscillation shifts from $0.31 \mathrm{~nm}^{-1}$ between 1000 and $2000 \mathrm{~s}$ towards $0.32 \mathrm{~nm}^{-1}$ at $3500 \mathrm{~s}$ (Fig S6). We attribute these oscillations to the bending of the NPLs which depart from their flat geometry to yield tubes or scrolls as was shown previously. ${ }^{12-14}$

To confirm this hypothesis, we computed the form factor of curved platelets with reasonable dimensions ${ }^{44}$ (see supplementary information for the calculation details) and noticed that indeed, marked oscillations appear in the $0.1-1 \mathrm{~nm}^{-1}$ region of the SAXS pattern when the radius of curvature is low enough. For a $30 \times 60 \mathrm{~nm}$ plate, oscillations start to appear when the radius of curvature goes below $20 \mathrm{~nm}$. Figure 5 shows a fit of the last SAXS pattern of the sequence with a model of curved NPL (see SI for details of the model). The high-q part of the experimental pattern is very well reproduced and the series of oscillations is also well described. However, a discrepancy is still present at lower wave-vectors. Our theoretical calculation also shows that the position of the oscillations is strongly dependent on the radius of curvature (see supplementary information): the first maximum shifts towards larger $q$ as the curvature radius decreases. The measured shift of the first oscillation maximum from $0.31 \mathrm{~nm}^{-1}$ to $0.32 \mathrm{~nm}^{-1}$ corresponds to a decrease of the NPL radius of curvature from 14 $\mathrm{nm}$ to $12 \mathrm{~nm}$. These values are close to the ones previously measured by TEM on large CdSe tubes and scrolls. ${ }^{13,45}$ Recently synthesized zinc blende CdSe nanotubes, though obtained through a slightly different chemical route, display similar curvatures. ${ }^{46}$

We wondered if our data could indicate whether the growth of the NPLs was concomitant to their bending or if the particle first grow and then bend after some time. To do so, we computed the form factor of nanoplaletets with constant lateral dimensions and varying radiuses of curvature (Figure S3). As the radius of curvature decreases while other param- 
eters are kept constant, the oscillations appear and the first minimum of the oscillations shifts towards larger wave-vectors. The value of the radius of curvature $R$ has an important effect on the position of the oscillation with positive shifts of more than $0.1 \mathrm{~nm}^{-1}$ for a 4 $\mathrm{nm}$ difference in radius. For a given curvature and increasing NPLs width, the oscillations shifts but to a much smaller extent (Figures S4). In the case of a bending with roughly constant lateral dimensions, we would thus observe an important shift in the position of the oscillations with time. On the contrary our data demonstrates that the most prominent temporal evolution of the oscillations is an increase in amplitude, rather than a positive shift towards higher $q$ values. Hence, our data qualitatively indicate that the NPLs develop in a bent fashion at a constant radius of curvature during the entire growth process and that the oscillations appear when the lateral dimension are sufficiently large compared to the radius of curvature. The fact that the NPLs grow curved and that the radius of curvature found in situ and in solution are the same than as those measured using TEM post synthesis and in vacuum is noteworthy. This points towards a spontaneous curvature of the NPLs which does not depend on the synthesis conditions but is encoded in the precise structure of the particle and the surface ligand.

We now turn to the interpretation of the low-q part of the SAXS patterns. As shown in figure 2.C and 4.B and as discussed previously, the intensity at low q shows an upturn after the $\mathrm{q}^{-2}$ regime when $\mathrm{q}$ decreases. Well separated non-interacting (curved) nanoplatelets would, in contrast, display a Guinier regime and ultimately a plateau at low-q as the one displayed by the theoretical models shown on figure 2.D. and in supplementary information in the case of curved NPLs. We discuss the possible origin of this discrepancy. The first possible explanation would be that the structure of the NPLs at large length scales does not correspond to the models we have hypothesized. Helicoidal structures for example, would give scattering patterns with a different behavior at small $\mathrm{q}$. In this case, the $\mathrm{q}^{-5}$ regime observed would not be a genuine power law regime. Another possible interpretation is the 


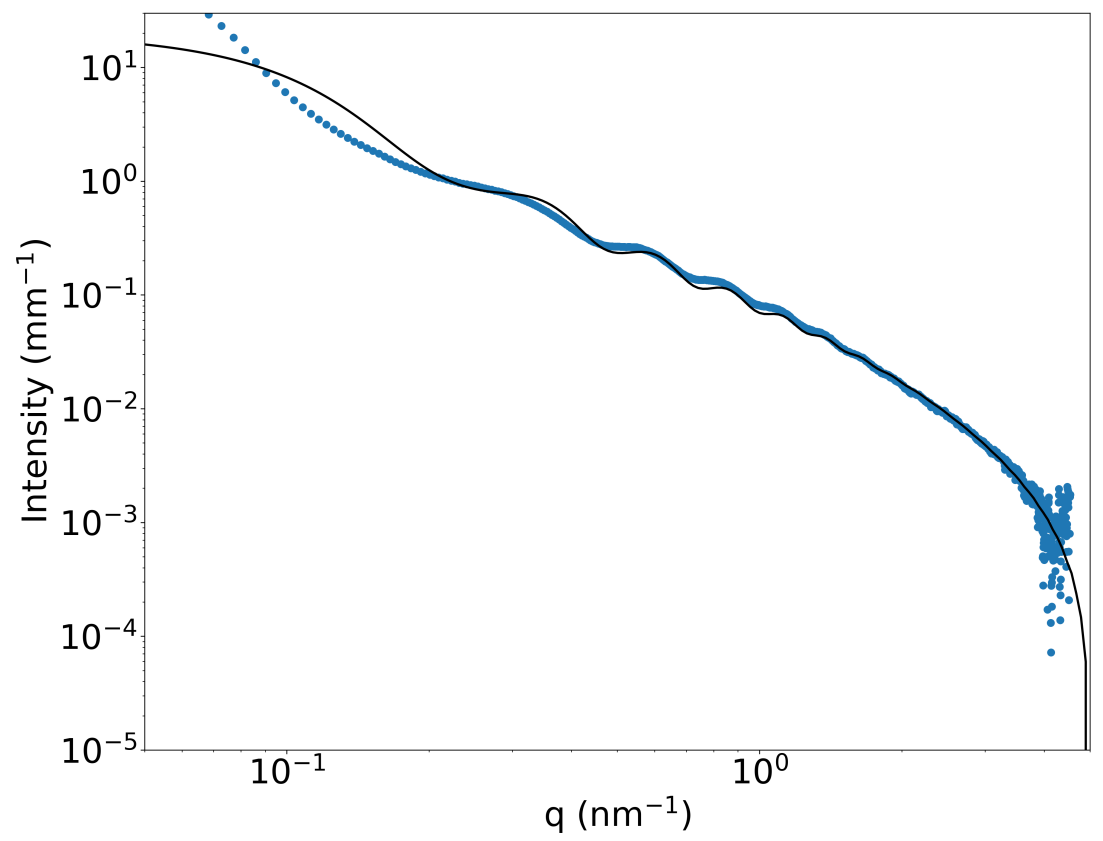

Figure 5: SAXS intensity at the end of the kinetic sequence at $2258 \mathrm{~s}$ and fit corresponding to a model of curved NPLs with a radius of curvature of $11.9 \mathrm{~nm}$, width of $43.0 \mathrm{~nm}$ and length of $45.3 \mathrm{~nm}$ with a polydispersity of $5 \%$ for each dimensions and a thickness of $0.9 \mathrm{~nm}$. A background corresponding to small micelles described by a Schulz distribution of sphere with mean radius $0.3 \mathrm{~nm}$ is also added (see SI for details). 
presence of attractive interaction between NPLs. An increase of the intensity at low $q$ is usually interpreted by the onset of a structure factor caused by inter-particle interactions. In the case of clays, which is another type of $2 \mathrm{D}$ nanoparticles, the effect of aggregation on the SAXS patterns has already been studied. Aggregation was induced by the addition of salt in aqueous dispersions and yielded low angle intensity versus q slopes comprised between 2 and 3.5. These slopes have been interpreted as the signature of fractal aggregates forming upon destabilization of the dispersion ${ }^{47}$ but exponents larger than 4 as observed here, can hardly be explained by aggregation. Such high slopes have been shown to appear in two instances: in the case of "fuzzy" interfaces where the variation of electron density between the two media is not abrupt but varies smoothly with the distance relative to the interface. Such a case has, for example, been observed in polymer systems. ${ }^{48}$ Another instance is the so-called Kirste-Porod scattering regime ${ }^{49,50}$ in which the scattered intensity decreases faster than $\mathrm{q}^{-4}$ due to the interplay between the mean and the Gaussien curvature of the interface in the asymptotic evaluation of I(q). Highly tortuous or convoluted interfaces originate a term proportional to $\mathrm{q}^{-6}$ in the scattered intensity. Though it is not possible to unequivocally assign one of these explanations to our results, we can safely state that the low-q behaviour of the scattered intensity points towards the presence of large aggregates of NPLs whose interface with the electron poor solvent is not sharp.

In conclusion, we have probed in situ using time-resolved SAXS-WAXS the formation mechanism of CdSe NPLs. Our data rule out unequivocally a templating effect or an oriented attachment formation mechanism where a large number of small nuclei assemble in an anisotropic fashion to yield the plate-like geometry. However, the evolution of the SAXS patterns, where a $\mathrm{q}^{-2}$ regime is present at early stages and extends towards smaller wavevectors with time is consistent with the gradual increase of the lateral extension of the NPLs from a small number of initial seeds. The WAXS patterns are consistent with this scenario since typical (220) peak shapes already observed for NPLs are confirmed during the particle 
formation in our experiments. We thus prove unambiguously that CdSe NPL form through the lateral growth of small nuclei. This agrees well with previous work which used complimentary UV-VIS probe ${ }^{15}$ and it is in line with the kinetic instability mechanism recently proposed. ${ }^{18}$ We also show that for a final temperature of $170^{\circ} \mathrm{C}$, the lateral extension is higher and provide experimental evidence that the NPL grow in a curved fashion. Finally, we proposed several possible explanations for the increase of the SAXS intensity at small wave-vectors. Further experiments probing larger length scales in the $100 \mathrm{~nm}-1 \mu \mathrm{m}$ regime are needed to clarify this issue.

\section{References}

(1) Ithurria, S.; Dubertret, B. Quasi 2D colloidal CdSe platelets with thicknesses controlled at the atomic level. J. Am. Chem. Soc. 2008, 130, 16504-5.

(2) Ithurria, S.; Tessier, M. D.; Mahler, B.; Lobo, R. P. S. M.; Dubertret, B.; Efros, A. L. Colloidal Nanoplatelets with Two-Dimensional Electronic Structure. Nat. Mater. 2011, 10, 936-41.

(3) Tessier, M. D.; Javaux, C.; Maksimovic, I.; Loriette, V.; Dubertret, B. Spectroscopy of Single CdSe Nanoplatelets. ACS Nano 2012, 6, 6751-6758.

(4) Rowland, C. E.; Fedin, I.; Zhang, H.; Gray, S. K.; Govorov, A. O.; Talapin, D. V.; Schaller, R. D. Picosecond energy transfer and multiexciton transfer outpaces Auger recombination in binary CdSe nanoplatelet solids. Nat. Mater. 2015, 14, 484-489.

(5) Abécassis, B.; Tessier, M. D.; Davidson, P.; Dubertret, B. Self-Assembly of CdSe Nanoplatelets into Giant Micrometer-Scale Needles Emitting Polarized Light. Nano Lett. 2014, 14, 710-715.

(6) Scott, R.; Heckmann, J.; Prudnikau, A. V.; Antanovich, A.; Mikhailov, A.; Owschimikow, N.; Artemyev, M.; Climente, J. I.; Woggon, U.; Grosse, N. B.; 
Achtstein, A. W. Directed emission of CdSe nanoplatelets originating from strongly anisotropic 2D electronic structure. Nat. Nanotechnol. 2017, 12, 1155-1160.

(7) Ma, X.; Diroll, B. T.; Cho, W.; Fedin, I.; Schaller, R. D.; Talapin, D. V.; Wiederrecht, G. P. Anisotropic Photoluminescence from Isotropic Optical Transition Dipoles in Semiconductor Nanoplatelets. Nano Lett. 2018, 18, 4647-4652.

(8) Mahler, B.; Nadal, B.; Bouet, C.; Patriarche, G.; Dubertret, B. Core/Shell Colloidal Semiconductor Nanoplatelets. J. Am. Chem. Soc. 2012, 134, 18591-18598.

(9) Pedetti, S.; Ithurria, S.; Heuclin, H.; Patriarche, G.; Dubertret, B. Type-II CdSe/CdTe Core/Crown Semiconductor Nanoplatelets. J. Am. Chem. Soc. 2014, 136, 16430-16438.

(10) Christodoulou, S.; Climente, J. I.; Planelles, J.; Brescia, R.; Prato, M.; MartínGarcía, B.; Khan, A. H.; Moreels, I. Chloride-Induced Thickness Control in CdSe Nanoplatelets. Nano Lett. 2018, 18, 6248-6254.

(11) Cho, W.; Kim, S.; Coropceanu, I.; Srivastava, V.; Diroll, B. T.; Hazarika, A.; Fedin, I.; Galli, G.; Schaller, R. D.; Talapin, D. V. Direct Synthesis of Six-Monolayer (1.9 Nm) Thick Zinc-Blende CdSe Nanoplatelets Emitting at 585 Nm. Chem. Mater. 2018, 30, 6957-6960.

(12) Bouet, C.; Mahler, B.; Nadal, B.; Abecassis, B.; Tessier, M. D.; Ithurria, S.; Xu, X.; Dubertret, B. Two-Dimensional Growth of CdSe Nanocrystals, from Nanoplatelets to Nanosheets. Chem. Mater. 2013, 25, 639-645.

(13) Hutter, E. M.; Bladt, E.; Goris, B.; Pietra, F.; van der Bok, J. C.; Boneschanscher, M. P.; de Mello Donegá, C.; Bals, S.; Vanmaekelbergh, D. Conformal and Atomic Characterization of Ultrathin CdSe Platelets with a Helical Shape. Nano Lett. 2014, 14, 6257-6262. 
(14) Jana, S.; de Frutos, M.; Davidson, P.; Abécassis, B. Ligand-induced twisting of nanoplatelets and their self-assembly into chiral ribbons. Sci. Adv. 2017, 3, e1701483.

(15) Ithurria, S.; Bousquet, G.; Dubertret, B. Continuous Transition from 3D to 1D Confinement Observed during the Formation of CdSe Nanoplatelets. J. Am. Chem. Soc. 2011, 133, 3070-3077.

(16) Son, J. et al. Large-Scale Soft Colloidal Template Synthesis of $1.4 \mathrm{~nm}$ Thick CdSe Nanosheets. Angew. Chem. Int. Ed. 2009, 48, 6861-6864.

(17) Son, J. S.; Yu, J. H.; Kwon, S. G.; Lee, J.; Joo, J.; Hyeon, T. Colloidal Synthesis of Ultrathin Two-Dimensional Semiconductor Nanocrystals. Adv. Mater. 2011, 23, 32143219.

(18) Riedinger, A.; Ott, F. D.; Mule, A.; Mazzotti, S.; Knüsel, P. N.; Kress, S. J. P.; Prins, F.; Erwin, S. C.; Norris, D. J. An intrinsic growth instability in isotropic materials leads to quasi-two-dimensional nanoplatelets. Nat. Mater. 2017, 16, 743-748.

(19) Schliehe, C.; Juarez, B. H.; Pelletier, M.; Jander, S.; Greshnykh, D.; Nagel, M.; Meyer, A.; Foerster, S.; Kornowski, A.; Klinke, C.; Weller, H. Ultrathin PbS Sheets by Two-Dimensional Oriented Attachment. Science 2010, 329, 550-553.

(20) Chen, Y.; Chen, D.; Li, Z.; Peng, X. Symmetry-Breaking for Formation of Rectangular CdSe Two-Dimensional Nanocrystals in Zinc-Blende Structure. J. Am. Chem. Soc. 2017, 139, 10009-10019.

(21) Liu, Y.; Zhang, B.; Fan, H.; Rowell, N.; Willis, M.; Zheng, X.; Che, R.; Han, S.; Yu, K. Colloidal CdSe 0-Dimension Nanocrystals and Their Self-Assembled 2-Dimension Structures. Chem. Mater. 2018, 30, 1575-1584.

(22) Ott, F. D.; Riedinger, A.; Ochsenbein, D. R.; Knüsel, P. N.; Erwin, S. C.; Mazzotti, M.; 
Norris, D. J. Ripening of Semiconductor Nanoplatelets. Nano Lett. 2017, 17, 68706877.

(23) Jiang, Y.; Ojo, W.-S.; Mahler, B.; Xu, X.; Abécassis, B.; Dubertret, B. Synthesis of CdSe Nanoplatelets without Short-Chain Ligands: Implication for Their Growth Mechanisms. ACS Omega 2018, 3, 6199-6205.

(24) Li, T.; Senesi, A. J.; Lee, B. Small Angle X-ray Scattering for Nanoparticle Research. Chem. Rev. 2016, 116, 11128-11180.

(25) Guinier, A.; Fournet, G. Small-angle scattering of X-rays; Wiley, 1955.

(26) Sun, Y.; Ren, Y. In Situ Synchrotron X-Ray Techniques for Real-Time Probing of Colloidal Nanoparticle Synthesis. Part. Part. Syst. Char. 2013, 30, 399-419.

(27) Pontoni, D.; Narayanan, T.; Rennie, A. R. Time-Resolved SAXS Study of Nucleation and Growth of Silica Colloids. Langmuir 2002, 18, 56-59.

(28) Abécassis, B.; Testard, F.; Spalla, O.; Barboux, P. Probing in situ the nucleation and growth of gold nanoparticles by small-angle X-ray scattering. Nano Lett. 2007, 7, 17231727.

(29) Abécassis, B.; Testard, F.; Kong, Q.; Francois, B.; Spalla, O. Influence of monomer feeding on a fast gold nanoparticles synthesis: time-resolved XANES and SAXS experiments. Langmuir 2010, 26, 13847-54.

(30) Abécassis, B.; Testard, F.; Spalla, O. Gold Nanoparticle Superlattice Crystallization Probed In Situ. Phys. Rev. Lett. 2008, 100, 115504.

(31) Sun, Y.; Zuo, X.; Sankaranarayanan, S. K. R. S.; Peng, S.; Narayanan, B.; Kamath, G. Quantitative 3D evolution of colloidal nanoparticle oxidation in solution. Science $\mathbf{2 0 1 7}$, 356, 303-307. 
(32) Golubkov, V.; Ekimov, A. I.; Onushchenko, A. A.; Tsekhomskii, V. Growth kinetics of $\mathrm{CuCl}$ microcrystals in a glassy matrix. Fizika i Khimiya Stekla 1981, 7, 397-401.

(33) Mattoussi, H.; Cumming, A. W.; Murray, C. B.; Bawendi, M. G.; Ober, R. Characterization of CdSe nanocrystallite dispersions by small angle x-ray scattering. J. Chem. Phys. 1996, 105, 9890-9896.

(34) Mattoussi, H.; Cumming, A. W.; Murray, C. B.; Bawendi, M. G.; Ober, R. Properties of CdSe nanocrystal dispersions in the dilute regime: Structure and interparticle interactions. Phys. Rev. B. 1998, 58, 7850-7863.

(35) Maes, J.; Castro, N.; De Nolf, K.; Walravens, W.; Abécassis, B.; Hens, Z. Size and Concentration Determination of Colloidal Nanocrystals by Small-Angle X-ray Scattering. Chem. Mater. 2018, 30, 3952-3962.

(36) Abécassis, B.; Bouet, C.; Garnero, C.; Constantin, D.; Lequeux, N.; Ithurria, S.; Dubertret, B.; Pauw, B. R.; Pontoni, D. Real-Time in Situ Probing of High-Temperature Quantum Dots Solution Synthesis. Nano Lett. 2015, 15, 2620-2626.

(37) van der Stam, W.; Rabouw, F. T.; Geuchies, J. J.; Berends, A. C.; Hinterding, S. O. M.; Geitenbeek, R. G.; van der Lit, J.; Prévost, S.; Petukhov, A. V.; de Mello Donega, C. In Situ Probing of Stack-Templated Growth of Ultrathin $\mathrm{Cu}{ }_{2}{ }_{x}$ S Nanosheets. Chem. Mater. 2016, 28, 6381-6389.

(38) Narayanan, T.; Sztucki, M.; Van Vaerenbergh, P.; Léonardon, J.; Gorini, J.; Claustre, L.; Sever, F.; Morse, J.; Boesecke, P. A Multipurpose Instrument for Time-Resolved Ultra-Small-Angle and Coherent X-Ray Scattering. J. Appl. Crystallogr. 2018, 51, $1511-1524$.

(39) Als-Nielsen, J.; McMorrow, D. Elements of Modern X-Ray Physics; Wiley: New York, NY, 2001. 
(40) Li, Z.; Peng, X. Size/Shape-Controlled Synthesis of Colloidal CdSe Quantum Disks: Ligand and Temperature Effects. J. Am. Chem. Soc. 2011, 133, 6578-6586.

(41) Chen, D.; Gao, Y.; Chen, Y.; Ren, Y.; Peng, X. Structure Identification of TwoDimensional Colloidal Semiconductor Nanocrystals with Atomic Flat Basal Planes. Nano Lett. 2015, 15, 4477-4482.

(42) Antanovich, A.; W. Achtstein, A.; Matsukovich, A.; Prudnikau, A.; Bhaskar, P.; Gurin, V.; Molinari, M.; Artemyev, M. A Strain-Induced Exciton Transition Energy Shift in CdSe Nanoplatelets: The Impact of an Organic Ligand Shell. Nanoscale 2017, 9, 18042-18053.

(43) Dufour, M.; Qu, J.; Greboval, C.; Méthivier, C.; Lhuillier, E.; Ithurria, S. Halide Ligands To Release Strain in Cadmium Chalcogenide Nanoplatelets and Achieve High Brightness. ACS Nano 2019, 13, 5326-5334.

(44) Constantin, D. Solution scattering from colloidal curved plates: barrel tiles, scrolls and spherical patches. J. Appl. Crystallogr. 2015, 48, 1901-1906.

(45) Bouet, C.; Tessier, M. D.; Ithurria, S.; Mahler, B.; Nadal, B.; Dubertret, B. Flat Colloidal Semiconductor Nanoplatelets. Chem. Mater. 2013, 25, 1262-1271.

(46) Huang, X.; Parashar, V. K.; Gijs, M. A. M. Spontaneous Formation of CdSe Photoluminescent Nanotubes with Visible-Light Photocatalytic Performance. ACS Cent. Sci. 2019, 5, 1017-1023.

(47) Michot, L. J.; Bihannic, I.; Thomas, F.; Lartiges, B. S.; Waldvogel, Y.; Caillet, C.; Thieme, J.; Funari, S. S.; Levitz, P. Coagulation of Na-Montmorillonite by Inorganic Cations at Neutral pH. A Combined Transmission X-ray Microscopy, Small Angle and Wide Angle X-ray Scattering Study. Langmuir 2013, 29, 3500-3510. 
(48) Wong, P.-z.; Bray, A. J. Porod scattering from fractal surfaces. Phys. Rev. Lett. 1988, $60,1344-1344$.

(49) Kirste, R.; Porod, G. Röntgenkleinwinkelstreuung an kolloiden Systemen Asymptotisches Verhalten der Streukurven. Kolloid Z Z Polym 1962, 184, 1-7.

(50) Bentley, P. M.; Cywinski, R. Evidence for a Spin Emulsion. Phys. Rev. Lett. 2008, 101, 227202.

\section{Acknowledgement}

We thank the ESRF for provision of the beam-time and Laurent Michot is acknowledged for discussions.

\section{Supporting Information Available}

Supplementary experimental details, SAXS figures, description of the theoretical model for bent nanoplatelets are provided in supplementary information. 


\section{For TOC only}
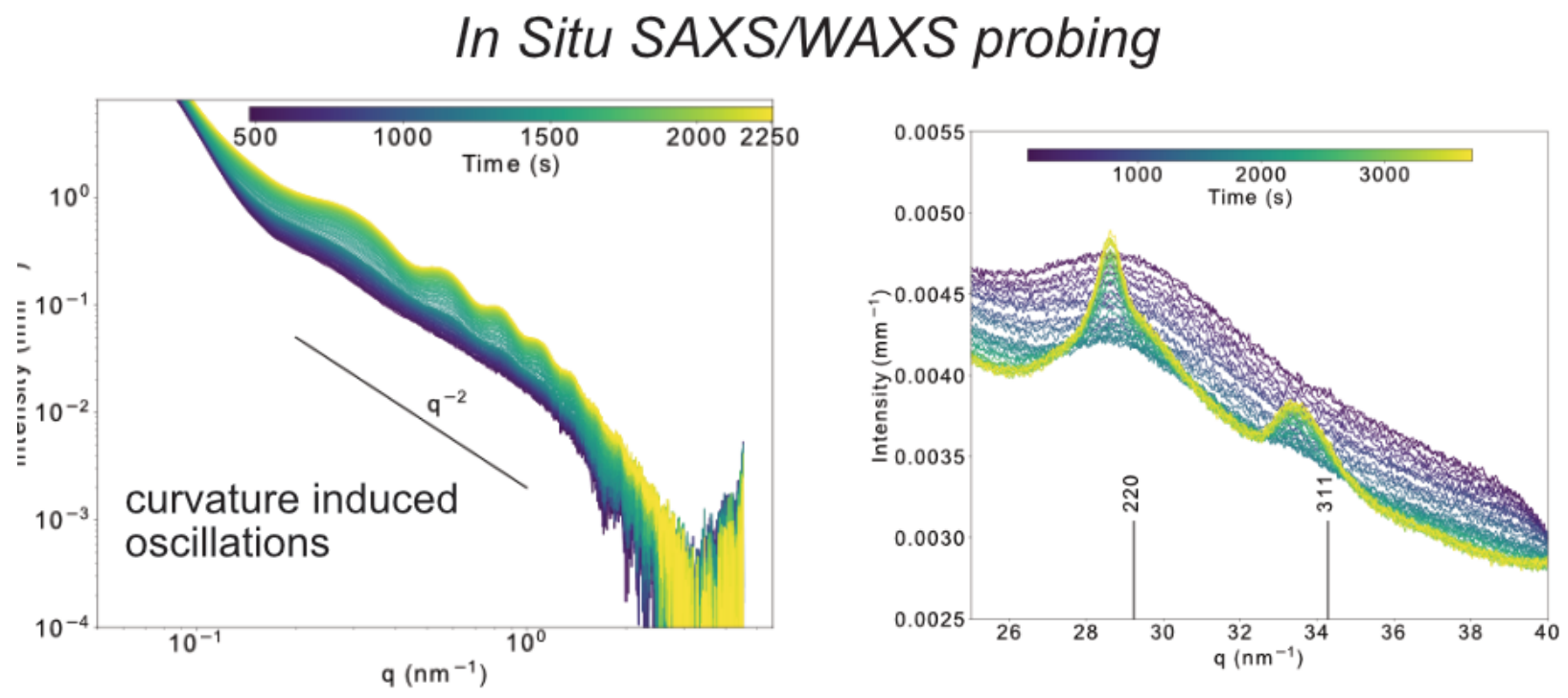